\title{
Networked Non-fragile Hळ Control for Lipschitz Nonlinear System with Quantization and Packet Dropout in Both Feedback and Forward Channels
}

\author{
Weiguo Ma, Xia Xu and Hairong Zhu \\ College of Electrical Engineering, Nantong University, Nantong, China
}

\begin{abstract}
The networked non-fragile Hळo control problem for Lipschitz nonlinear system with quantization and packet dropout in both feedback and forward channels is investigated in this paper. The sensor measurement and controller output are quantized by logarithmic quantizers before beeing transmitted over the network. The packet transmissions in the communication channels from the sensor to the controller and from the controller to the actuator are modeled as Markov chains respectively. Based on the Lyapunov functional approach, the networked non-fragile $\mathrm{H} \infty$ controller is designed to stabilize the Lipschitz nonlinear system and achieve the prescribed $\mathrm{H} \infty$ performance. Finally, a numerical example is provided to illustrate the effectiveness and superiority of the proposed method.
\end{abstract}

ACM CCS (2012) Classification: Applied computing $\rightarrow$ Operations research $\rightarrow$ Industry and manufacturing $\rightarrow$ Command and control

Information systems $\rightarrow$ Information systems applications $\rightarrow$ Process control systems

Keywords: non-fragile Hळ control, Lipschitz nonlinear system, quantization, packet dropout

\section{Introduction}

Networked control system where the control loop is closed through communication network has attracted considerable attention in recent years. Networked control system has been applied in a broad range of areas such as complicated industrial control system, remote control of robot, unmanned aircrafts and vehicles, etc
[1]. In networked control system, the sensors, controllers and actuators exchange information via the shared communication network. The introduction of network in control loop gives rise to some issues such as data quantization [2], [3], network induced delay [4] and packet dropout [5] - [8], which complicate the analysis and design of networked control systems.

There exists perturbation in the coefficient of the controller due to the effect of finite resolution measuring instruments, finite word length and roundoff errors [9]. Therefore, it is necessary that the non-fragile controller is designed. Many efforts have been made in networked control system recently. The network induced delay is transformed into the uncertainties of the system coefficients for networked control system with network induced delay. Then nonfragile controllers are designed based on robust control theory [10] - [12]. In [13], the networked control system is modeled as time delay system, and then the non-fragile guaranteed cost fault tolerant controller is designed. Using the structure vertex separator method, the problem of non-fragile $\mathrm{H} \infty$ control is studied for networked continuous time linear system with time delay and logarithmic quantizer in [14]. Considering the randomly occurring gain variations, distributed delays and channel fadings, Li et al. designed the non-fragile $\mathrm{H} \infty$ controller for networked discrete time linear system [15] $-[16]$. For networked switch fuzzy time delay 
system, Liu and Sun design the non-fragile controller based on parallel distributed compensation and multiple Lyapunov functions method [17]. It is worth pointing out that the existing literature is concerned with non-fragile control of networked control system with quantization and/or network induced delay. To the best of the authors' knowledge, the problem of networked non-fragile $\mathrm{H} \infty$ control for Lipschitz nonlinear system subject to quantization and packet dropout, both in the feedback and forward paths, has not been fully investigated, which motivates the present study.

The rest of this paper is organized as follows. In Section 2, the effects of quantization and packet dropout are analyzed. The networked control system is modeled as a Markovian jumping system. The sufficient condition for the existence of non-fragile $\mathrm{H} \infty$ controller is given in terms of linear matrix inequality in Section 3. A simulation example is presented to illustrate the effectiveness of the proposed method in Section 4. In Section 5, conclusions are given.

Notation: $\mathbf{R}^{n}$ denotes the $n$ dimensional Euclidean space. || || stands for the Euclidean norm. $I$ and 0 denote the identity and zero matrices of compatible dimensions respectively. $\operatorname{Pr}[\cdot]$ means the occurrence probability of event ".". $E\{x\}$ stands for the expectation of stochastic variable $x . M^{T}$ and $M^{-1}$ denote respectively the transpose and inverse of a matrix $M . X>0$ $(X<0)$ means that the matrix $X$ is real symmetric positive definite (negative definite). The asterisk "*" in a matrix is used to represent the term that is induced by symmetry. $\operatorname{diag}\left\{M_{1}, \ldots\right.$, $M_{2}$ \} stands for a block-diagonal matrix with the matrices $M_{1}, \ldots, M_{2}$ on the diagonal.

\section{Problem Formulation and Preliminaries}

The diagram of networked control for Lipschitz nonlinear system studied in this paper is shown in Figure 1. Lipschitz nonlinear system is very common. For example, the sinusoidal terms encountered in some problems of robotics are global Lipschitz. Considered in a given neighborhood, most nonlinearities are local Lipschitz [18].

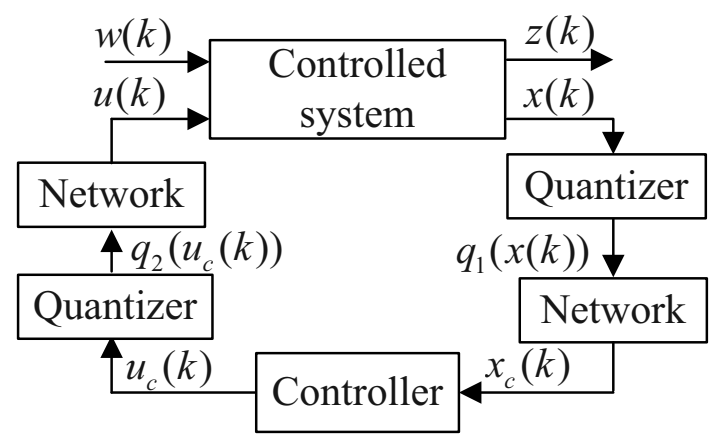

Figure 1. Diagram of control for networked nonlinear system.

The controlled system is described by

$$
\left\{\begin{aligned}
x(k+1) & =A x(k)+B_{1} u(k)+B_{2} w(k) \\
& +F f(k, x(k)) \\
z(k) & =C x(k)+D w(k)
\end{aligned}\right.
$$

where $x(k) \in \mathbf{R}^{n}$ is the state, $u(k) \in \mathbf{R}^{p}$ is the control input, $w(k) \in \mathbf{R}^{m}$ is the disturbance belonging to $L_{2}[0, \infty], z(k) \in \mathbf{R}^{q}$ is the controlled output. $A, B_{1}, B_{2}, F, C$ and $D$ are known constant matrices with appropriate dimensions. $f(k, x(k))$ is a nonlinear vector function that is assumed to be Lipschitz with respect to $x(k)$, i.e. there exists known constant matrix $F_{1}$ such that $f(0,0)=0,\left\|f\left(k, x_{1}(k)\right)-f\left(k, x_{2}(k)\right)\right\| \leq$ $\left\|F_{1}\left(x_{1}(k)-x_{2}(k)\right)\right\|$.

The system state is quantized before beeing transmitted to the controller via the network due to the limited bandwidth. Logarithmic quantizers are essential for quadratic stabilization via quantized feedback if a coarse quantization density is required. Nonlogarithmic quantizers such as finite quantizers and linear quantizers are unsuitable [19]. The following logarithmic quantizer is used in this paper

$$
q_{1}(v)= \begin{cases}u_{i}, & \text { if } u_{i} /(1+\delta)<v \leq \\ 0, & u_{i} /(1-\delta), v>0 \\ -q_{1}(-v), & \text { if } v=0\end{cases}
$$

where the set of quantized levels $U=\left\{ \pm u_{i}, u_{i}=\right.$ $\left.\rho_{i} u_{0}, i= \pm 1, \pm 2, \ldots\right\} \cup\left\{ \pm u_{0}\right\} \cup\{0\}, 0<\rho<1$, $u_{0}>0, \delta=(1-\rho) /(1+\rho), \rho$ is the quantization density of $q_{1}(v)$. The quantized state can be described by 


$$
\begin{aligned}
q_{1}(x(k)) & =\left[\begin{array}{lll}
q_{1}\left(x_{1}(k)\right) & \cdots & q_{1}\left(x_{n}(k)\right)
\end{array}\right]^{T} \\
& =\left(I+H_{1}(k)\right) x(k)
\end{aligned}
$$

where $\left\|H_{1}(k)\right\| \leq \delta_{1}$.

When the quantized data are transmitted over the network, it is supposed that the network induced delay is small enough to be neglected. In addition, due to the disturbance or network congestion, the data packet may be dropped out, which is common in wireless network. The packet transmission process can be modeled as Markov chain [8]. Assume that Markov chains $\alpha(k)$ and $\beta(k)$ denote the packet transmission process in the channels from the sensor to the controller and from the controller to the actuator respectively. The finite state sets are $\Phi_{1}=$ $\{0,1\}$ and $\Phi_{2}=\{0,1\}$. The state 0 means that the data packet is dropped out. The state 1 indicates that the data packet is transmitted successfully. The transition probability matrices of Markov chains are $\bar{P}=\left[\lambda_{i j}\right]$ and $\bar{Q}=\left[\pi_{r s}\right]$ with

$\lambda_{i j}=\operatorname{Pr}[\alpha(k+1)=j \mid \alpha(k)=i], \lambda_{i j} \geq 0$,

$\sum_{j=0}^{1} \lambda_{i j}=1, \pi_{r s}=\operatorname{Pr}[\beta(k+1)=s \mid \beta(k)=r]$,

$\pi_{r s} \geq 0, \sum_{s=0}^{1} \pi_{r s}=1, \forall i, j \in \Phi_{1}, \forall r, s \in \Phi_{2}$.

The input of the controller can be described by

$$
\begin{aligned}
x_{c}(k)= & \alpha(k)\left(I+H_{1}(k)\right) x(k) \\
& +(1-\alpha(k)) x_{c}(k-1)
\end{aligned}
$$

It is necessary that the controller which is part of a closed-loop system can tolerate some uncertainty in its coefficients [9]. Consider the non-fragile controller

$$
u_{c}(k)=\left(K_{\alpha(k)}+\Delta K\right) x_{c}(k)
$$

where $K_{\alpha(k)}$ is the controller gain, $\Delta K$ stands for the controller gain perturbations which are of additive and multiplicative forms:

$$
\begin{gathered}
\Delta K=M_{2} F_{2}(k) N_{2}, \quad F_{2}^{T}(k) F_{2}(k) \leq I \\
\Delta K=M_{3} F_{3}(k) N_{3} K_{\alpha(k)}, \quad F_{3}^{T}(k) F_{3}(k) \leq I
\end{gathered}
$$

where $M_{2}, N_{2}, M_{3}, N_{3}$ are known constant matrices.
When the guided system is controlled, the controller $u_{c}(k)=V-\left(K_{\alpha(k)}+\Delta K\right) x_{c}(k)$, where $V$ is the reference signal.

The output of the controller $u_{c}(k)$ is also quantized by a logarithmic quantizer $q_{2}(v)$ which is similar to the state quantizer $q_{1}(v)$. The control input of the system is described by

$$
\begin{aligned}
u(k)= & \beta(k)\left(I+H_{2}(k)\right) u_{c}(k) \\
& +(1-\beta(k)) u(k-1)
\end{aligned}
$$

where $\left\|H_{2}(k)\right\| \leq \delta_{2}, \delta_{2}=\left(1-\rho_{2}\right) /\left(1+\rho_{2}\right), \rho_{2}$ is the quantization density of $q_{2}(v)$.

Defining the augmented state $\bar{x}(k)=\left[x^{T}(k)\right.$, $\left.x_{c}{ }^{T}(k-1), u^{T}(k-1)\right]^{T}$, the networked control system can be modeled as

$$
\left\{\begin{aligned}
\bar{x}(k+1) & =\bar{A}(\alpha(k), \beta(k)) \bar{x}(k)+\bar{B} w(k) \\
& +\bar{F} f(k, x(k)) \\
z(k) & =\bar{C} \bar{x}(k)+\bar{D} w(k)
\end{aligned}\right.
$$

where

$$
\begin{aligned}
\bar{A}(\alpha(k), \quad \beta(k))=\left[\begin{array}{lll}
\bar{A}_{11} & \bar{A}_{12} & \bar{A}_{13} \\
\bar{A}_{21} & \bar{A}_{22} & 0 \\
\bar{A}_{31} & \bar{A}_{32} & \bar{A}_{33}
\end{array}\right], \\
\bar{B}=\left[\begin{array}{c}
B_{2} \\
0 \\
0
\end{array}\right], \bar{F}=\left[\begin{array}{c}
F \\
0 \\
0
\end{array}\right], \bar{C}=[C, 0,0], \bar{D}=D, \\
\bar{A}_{11}=A+\beta(k) \alpha(k) B_{1}\left(I+H_{2}(k)\right)\left(K_{\alpha(k)}\right. \\
+\Delta K)\left(I+H_{1}(k)\right), \\
\bar{A}_{12}=\beta(k)(1-\alpha(k)) B_{1}\left(I+H_{2}(k)\right)\left(K_{\alpha(k)}+\Delta K\right), \\
\bar{A}_{13}=B_{1}(1-\beta(k)), \bar{A}_{21}=\alpha(k)\left(I+H_{1}(k)\right), \\
\bar{A}_{22}=(1-\alpha(k)) I, \\
\bar{A}_{31}=\beta(k) \alpha(k)\left(I+H_{2}(k)\right)\left(K_{\alpha(k)}+\Delta K\right) \\
\quad \cdot\left(I+H_{1}(k)\right), \\
\bar{A}_{32}=\beta(k)(1-\alpha(k))\left(I+H_{2}(k)\right)\left(K_{\alpha(k)}+\Delta K\right), \\
\bar{A}_{33}=(1-\beta(k)) I .
\end{aligned}
$$

Because the networked control system is modeled as Markovian jumping system, we introduce the following stochastically stable and $\mathrm{H} \infty$ performance definitions and a lemma which will be used in the sequel. 
Definition 1 [20]. When $w(k)=0$, the networked control system (9) is said to be stochastically stable if for every initial state $(\bar{x}(0), \alpha(0)$, $\beta(0)), E\left\{\sum_{k=0}^{\infty}\|\bar{x}(k)\|^{2}\right\}<\infty$.

Definition 2 [20]. Given a scalar $\gamma>0$, the networked control system (9) is said to have an $\mathrm{H} \infty$ performance $\gamma$, if

$\sum_{k=0}^{\infty} E\left\{z^{T}(k) z(k)\right\}<\gamma^{2} \sum_{k=0}^{\infty} E\left\{w^{T}(k) w(k)\right\}$

for all nonzero $w(k) \in \mathrm{L}_{2}[0, \infty\rangle$ under zero initial condition.

Lemma 1 [21]. Given matrices $\Gamma_{1}=\Gamma_{1}^{T}, \Gamma_{2}$ and $\Gamma_{3}$ of appropriate dimensions, then $\Gamma_{1}+\Gamma_{2} F_{4}(k)$ $\Gamma_{3}+\Gamma_{3}^{T} F_{4}^{T}(k) \Gamma_{2}<0$ holds for all $F_{4}(k)$ satisfying $F_{4}^{T}(k) F_{4}(k) \leq I$, if and only if there exists scalar $\varepsilon>0$ such that $\Gamma_{1}+\varepsilon^{-1} \Gamma_{2} \Gamma_{2}^{T}+\varepsilon \Gamma_{3}^{T} \Gamma_{3}<0$.

\section{Non-Fragile $\mathrm{H} \infty$ Controller Design}

In this section, we explore the sufficient condition of the existence of non-fragile $\mathrm{H} \infty$ controller for networked control system (9). First, we establish the sufficient condition of stability for the networked control system.

Theorem 1. Given the quantization densities $\rho_{1}, \rho_{2}$ and controller gain $K_{\alpha(k)}+\Delta K$, the networked control system (9) is stochastically stable if there exist symmetric positive definite matrices $P(i, r)=\operatorname{diag}\left\{P_{1}(i, r), P_{2}(i, r)\right.$, $\left.P_{3}(i, r)\right\}, i \in \Phi_{1}, r \in \Phi_{2}$, scalar $\varepsilon>0$ such that the following matrix inequalities hold

$$
\left[\begin{array}{ccc}
-P(i, r)+\varepsilon \Phi & * & * \\
0 & -\varepsilon I & * \\
\Xi_{31} & \Xi_{32} & \Xi_{33}
\end{array}\right]<0
$$

where

$$
\begin{aligned}
\Xi_{31}= & {\left[\bar{A}^{T}(i, r), \bar{A}^{T}(i, r), \bar{A}^{T}(i, r), \bar{A}^{T}(i, r)\right]^{T}, } \\
\Xi_{32}= & {\left[\bar{F}^{T}, \bar{F}^{T}, \bar{F}^{T}, \bar{F}^{T}\right]^{T}, } \\
\Xi_{33}= & \operatorname{diag}\left\{-1 /\left(\lambda_{i 0} \pi_{r 0}\right) P^{-1}(0,0),\right. \\
& -1 /\left(\lambda_{i 0} \pi_{r 1}\right) P^{-1}(0,1),-1 /\left(\lambda_{i 1} \pi_{r 0}\right) P^{-1}(1,0), \\
& \left.-1 /\left(\lambda_{i 1} \pi_{r 1}\right) P^{-1}(1,1)\right\},
\end{aligned}
$$

$\Phi=\left[\begin{array}{cc}F_{1}^{T} F_{1} & 0 \\ 0 & 0\end{array}\right]$

Proof. Consider the following Lyapunov functional $V[\bar{x}(k), \alpha(k)=i, \beta(k)=r]=\bar{x}^{T}(k) P(i, r) x(k)$ where $P(i, r)=\operatorname{diag}\left\{P_{1}(i, r), P_{2}(i, r), P_{3}(i, r)\right\}$, $P_{1}(i, r), P_{2}(i, r)$ and $P_{3}(i, r)$ are symmetric positive definite matrices.

As $f^{T}(k, x(k)) f(k, x(k)) \leq x^{T}(k) F_{1}^{T} F_{1} x(k)$, there exists $\varepsilon>0$ so that $\varepsilon x^{T}(k) F_{1}^{T} F_{1} x(k)-f^{T}(k, x(k))$ $f(k, x(k)) \geq 0$.

Taking the difference of $V[\bar{x}(k), \alpha(k)=i, \beta(k)=$ $r$ ] along the trajectory of system (9) with $w(k)$ $=0$, we can obtain

$$
\begin{aligned}
\Delta V(k)= & E\{V[\bar{x}(k+1), \alpha(k+1), \beta(k+1) \mid \bar{x}(k), \\
& \alpha(k)=i, \beta(k)=r]\}-V[\bar{x}(k), \alpha(k)=i, \\
& \beta(k)=r]=\zeta^{T}(k) \tilde{\Xi}(i, r) \zeta(k)
\end{aligned}
$$

where

$$
\begin{aligned}
& \tilde{\Xi}(i, r)=\left[\begin{array}{cc}
\tilde{\Xi}(i, r)_{11} & * \\
\bar{F}^{T} H \bar{A} & \bar{\Phi}^{T} H \bar{F}-\varepsilon I
\end{array}\right], \\
& \zeta(k)=\left[\bar{x}^{T}(k), f^{T}(k, x(k))\right]^{T}, \\
& \tilde{\Xi}(i, r)_{11}=\bar{A}^{T}(i, r) H \bar{A}(i, r)-P(i, r)+\varepsilon \Phi, \\
& H=\sum_{j=0}^{1} \sum_{s=0}^{1} \lambda_{i j} \pi_{r s} P(j, s) .
\end{aligned}
$$

Thus,

$$
\Delta V(k) \leq-\phi \zeta^{T}(k) \zeta(k) \leq-\phi \bar{x}^{T}(k) \bar{x}(k)
$$

where $\phi$ is the least eigenvalue of $-\tilde{\Xi}(i, r)$.

Then,

$E\left\{\sum_{k=0}^{\infty}\|\bar{x}(k)\|^{2}\right\}<\phi^{-1} \bar{x}^{T}(0) P(\alpha(0), \beta(0)) \bar{x}(0)<\infty$

Therefore, by Definition 1, the networked control system (9) is stochastically stable. The proof is completed.

The following theorem proposes the sufficient condition for the existence of non-fragile $\mathrm{H} \infty$ controller for networked control system (9).

Theorem 2. Given a scalar $\gamma>0$, quantization densities $\rho_{1}$ and $\rho_{2}$, the networked control system (9) is stochastically stable and has an Hळ performance $\gamma$, if there exist scalars $\varepsilon_{1}>$ 
$0, \varepsilon_{2}>0, \varepsilon_{3}>0, \varepsilon_{4}>0, \varepsilon_{5}>0, \varepsilon_{6}>0$, symmetric positive definite matrices $X(i, r)=\operatorname{diag}$ $\left\{X_{1}(i, r), X_{2}(i, r), X_{3}(i, r)\right\}, i \in \Phi_{1}, r \in \Phi_{2}, Q$, matrices $Y_{0}$ and $Y_{1}$ such that the following linear matrix inequalities hold

$$
\left[\begin{array}{cccc}
\Omega_{1,11} & * & * & * \\
\Omega_{1,21} & \Omega_{1,22} & * & * \\
\Omega_{1,31} & 0 & \Omega_{1,33} & * \\
\Omega_{1,41} & 0 & 0 & \Omega_{1,44}
\end{array}\right]<0
$$

$$
\left[\begin{array}{ccccc}
\Omega_{2,11} & * & * & * & * \\
\Omega_{2,21} & \Omega_{2,22} & * & * & * \\
\Omega_{2,31} & 0 & \Omega_{2,33} & * & * \\
\Omega_{2,41} & \Omega_{2,42} & 0 & \Omega_{2,44} & * \\
\Omega_{2,51} & \Omega_{2,52} & 0 & \Omega_{2,54} & \Omega_{2,55}
\end{array}\right]<0
$$

$$
\left[\begin{array}{cccc}
\Omega_{3,11} & * & * & * \\
\Omega_{3,21} & \Omega_{3,22} & * & * \\
\Omega_{3,31} & 0 & \Omega_{3,33} & * \\
\Omega_{3,41} & \Omega_{3,42} & 0 & \Omega_{3,44}
\end{array}\right]<0
$$

$$
\left[\begin{array}{ccccc}
\Omega_{4,11} & * & * & * & * \\
\Omega_{4,21} & \Omega_{4,22} & * & * & * \\
\Omega_{4,31} & \Omega_{4,32} & \Omega_{4,33} & * & * \\
\Omega_{4,41} & \Omega_{4,42} & \Omega_{4,43} & \Omega_{4,44} & * \\
\Omega_{4,51} & \Omega_{4,52} & \Omega_{4,53} & \Omega_{4,54} & \Omega_{4,55}
\end{array}\right]<0
$$

where $\Omega_{1,11}=\operatorname{diag}\left\{-X(0,0),-\gamma^{2} I,-\varepsilon_{1} I\right\}$,

$$
\Omega_{1,21}=\left[\begin{array}{lll}
\bar{A}(0,0) X(0,0) & \bar{B} & \varepsilon_{1} \bar{F} \\
\bar{A}(0,0) X(0,0) & \bar{B} & \varepsilon_{1} \bar{F} \\
\bar{A}(0,0) X(0,0) & \bar{B} & \varepsilon_{1} \bar{F} \\
\bar{A}(0,0) X(0,0) & \bar{B} & \varepsilon_{1} \bar{F}
\end{array}\right]
$$

$\Omega_{1,22}=\operatorname{diag}\left\{-1 /\left(\lambda_{00} \pi_{00}\right) X(0,0),-1 /\left(\lambda_{00} \pi_{01}\right) X(0,1)\right.$, $\left.-1 /\left(\lambda_{01} \pi_{00}\right) X(1,0),-1 /\left(\lambda_{01} \pi_{01}\right) X(1,1)\right\}, \Omega_{1,31}=$ $[\bar{C}, \bar{D}, 0], \Omega_{1,33}=-I, \Omega_{1,41}=\left[\bar{F}_{1}, X(0,0), 0,0\right], \bar{F}_{1}$ $=\left[F_{1}, 0,0\right], \Omega_{1,44}=-\varepsilon_{1} I, \Omega_{2,11}=\operatorname{diag}\{-X(0,1)$, $\left.-\gamma^{2} I,-\varepsilon_{1} I\right\}$,
$\Omega_{2,21}=\left[\begin{array}{lll}\Pi_{2,21} & \bar{B} & \varepsilon_{1} \bar{F} \\ \Pi_{2,21} & \bar{B} & \varepsilon_{1} \bar{F} \\ \Pi_{2,21} & \bar{B} & \varepsilon_{1} \bar{F} \\ \Pi_{2,21} & \bar{B} & \varepsilon_{1} \bar{F}\end{array}\right]$,

$\Pi_{2,21}=\left[\begin{array}{ccc}A X_{1}(0,1) & B_{1} Y_{0} & 0 \\ 0 & X_{2}(0,1) & 0 \\ 0 & Y_{0} & 0\end{array}\right]$,

$\Omega_{2,22}=\operatorname{diag}\left\{-1 /\left(\lambda_{00} \pi_{10}\right) X(0,0),-1 /\left(\lambda_{00} \pi_{11}\right) X(0,1)\right.$,

$\left.-1 /\left(\lambda_{01} \pi_{10}\right) X(1,0),-1 /\left(\lambda_{01} \pi_{11}\right) X(1,1)\right\}$,

$\Omega_{2,31}=\left[\begin{array}{lllll}C X_{1}(0,1) & 0 & 0 & D & 0 \\ F_{1} X_{1}(0,1) & 0 & 0 & 0 & 0\end{array}\right]$

$\Omega_{2,33}=\operatorname{diag}\left\{-I,-\varepsilon_{1} I\right\}$,

$\Omega_{2,41}=\left[\begin{array}{ccccc}0 & 0 & 0 & 0 & 0 \\ 0 & N_{2} X_{2}(0,1) & 0 & 0 & 0\end{array}\right]$,

$\Omega_{2,42}=\left[\begin{array}{llll}\Pi_{2,42} & \Pi_{2,42} & \Pi_{2,42} & \Pi_{2,42}\end{array}\right]$,

$\Pi_{2,42}=\left[\begin{array}{ccc}\varepsilon_{2} M_{2}^{T} B_{1}^{T} & 0 & \varepsilon_{2} M_{2}^{T} \\ 0 & 0 & 0\end{array}\right]$,

$\Omega_{2,44}=\operatorname{diag}\left\{-\varepsilon_{2} I,-\varepsilon_{2} I\right\}$,

$\Omega_{2,51}=\left[\begin{array}{ccccc}0 & 0 & 0 & 0 & 0 \\ 0 & Y_{0} & 0 & 0 & 0\end{array}\right]$,

$\Omega_{2,52}=\left[\Pi_{2,52}, \Pi_{2,52}, \Pi_{2,52}, \Pi_{2,52}\right]$,

$\Pi_{2,52}=\left[\begin{array}{ccc}\varepsilon_{3} B_{1}^{T} & 0 & \varepsilon_{3} I \\ 0 & 0 & 0\end{array}\right]$,

$\Omega_{2,54}=\left[\begin{array}{cc}0 & 0 \\ 0 & \varepsilon_{2} M_{2}\end{array}\right]$,

$\Omega_{2,55}=\operatorname{diag}\left\{-\varepsilon_{3} I,-\varepsilon_{3} \delta_{2}^{2} I\right\}$,

$\Omega_{3,11}=\operatorname{diag}\left\{-X(1,0),-\gamma^{2} I,-\varepsilon_{1} I\right\}$,

$\Omega_{3,21}=\left[\begin{array}{lll}\Pi_{3,21} & \bar{B} & \varepsilon_{1} \bar{F} \\ \Pi_{3,21} & \bar{B} & \varepsilon_{1} \bar{F} \\ \Pi_{3,21} & \bar{B} & \varepsilon_{1} \bar{F} \\ \Pi_{3,21} & \bar{B} & \varepsilon_{1} \bar{F}\end{array}\right]$, 
$\Pi_{3,21}=\left[\begin{array}{ccc}A X_{1}(1,0) & 0 & B_{1} X_{3}(1,0) \\ X_{1}(1,0) & 0 & 0 \\ 0 & 0 & X_{3}(1,0)\end{array}\right]$,

$\Omega_{3,22}=\operatorname{diag}\left\{-1 /\left(\lambda_{10} \pi_{00}\right) X(0,0),-1 /\left(\lambda_{10} \pi_{01}\right) X(0,1)\right.$,

$\left.-1 /\left(\lambda_{11} \pi_{00}\right) X(1,0),-1 /\left(\lambda_{11} \pi_{01}\right) X(1,1)\right\}$,

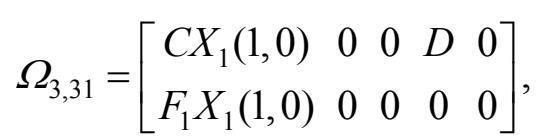

$\Omega_{3,33}=\Omega_{2,33}$,

$\Omega_{3,41}=\left[\begin{array}{ccccc}0 & 0 & 0 & 0 & 0 \\ X_{1}(1,0) & 0 & 0 & 0 & 0\end{array}\right]$,

$\Omega_{3,42}=\left[\Pi_{3,42}, \Pi_{3,42}, \Pi_{3,42}, \Pi_{3,42}\right]$,

$\Pi_{3,42}=\left[\begin{array}{ccc}0 & \varepsilon_{4} I & 0 \\ 0 & 0 & 0\end{array}\right]$,

$\Omega_{3,44}=\operatorname{diag}\left\{-\varepsilon_{4} I,-\varepsilon_{4} \delta_{1}^{2} I\right\}$,

$\Omega_{4,11}=\operatorname{diag}\left\{-X(1,1),-\gamma^{2} I,-\varepsilon_{1} I\right\}$

$+\operatorname{diag}\left\{\delta_{1}^{2} Q, 0,0\right\}$,

$\Omega_{4,21}=\left[\begin{array}{lll}\Pi_{4,21} & \bar{B} & \varepsilon_{1} \bar{F} \\ \Pi_{4,21} & \bar{B} & \varepsilon_{1} \bar{F} \\ \Pi_{4,21} & \bar{B} & \varepsilon_{1} \bar{F} \\ \Pi_{4,21} & \bar{B} & \varepsilon_{1} \bar{F}\end{array}\right]$,

$\Pi_{4,21}=\left[\begin{array}{ccc}A X_{1}(1,1)+B_{1} Y_{1} & 0 & 0 \\ X_{1}(1,1) & 0 & 0 \\ Y_{1} & 0 & 0\end{array}\right]$,

$\Omega_{4,22}=\operatorname{diag}\left\{-1 /\left(\lambda_{10} \pi_{10}\right) X(0,0),-1 /\left(\lambda_{10} \pi_{11}\right) X(0,1)\right.$, $\left.-1 /\left(\lambda_{11} \pi_{10}\right) X(1,0),-1 /\left(\lambda_{11} \pi_{11}\right) X(1,1)\right\}$,

$\Omega_{4,31}=\left[\begin{array}{ccccc}C X_{1}(1,1) & 0 & 0 & D & 0 \\ F_{1} X_{1}(1,1) & 0 & 0 & 0 & 0 \\ 0 & 0 & 0 & 0 & 0\end{array}\right]$,

$\Omega_{4,32}=\left[\Pi_{4,32}, \Pi_{4,32}, \Pi_{4,32}, \Pi_{4,32}\right]$,

$\Pi_{4,32}=\left[\begin{array}{ccc}0 & 0 & 0 \\ 0 & 0 & 0 \\ Y_{1}^{T} B_{1}^{T} & I & Y_{1}^{T}\end{array}\right]$,
$\Omega_{4,33}=\operatorname{diag}\{-I,-\varepsilon I,-Q\}$,

$\Omega_{4,41}=\left[\begin{array}{ccccc}0 & 0 & 0 & 0 & 0 \\ N_{2} X_{1}(1,1) & 0 & 0 & 0 & 0\end{array}\right]$,

$\Omega_{4,42}=\left[\Pi_{4,42}, \Pi_{4,42}, \Pi_{4,42}, \Pi_{4,42}\right]$,

$\Pi_{4,42}=\left[\begin{array}{ccc}\varepsilon_{5} M_{2}^{T} B_{1}^{T} & 0 & \varepsilon_{5} M_{2}^{T} \\ 0 & 0 & 0\end{array}\right]$,

$\Omega_{4,43}=\left[\begin{array}{llc}0 & 0 & 0 \\ 0 & 0 & N_{2} X_{1}(1,1)\end{array}\right]$,

$\Omega_{4,44}=\operatorname{diag}\left\{-\varepsilon_{5} I,-\varepsilon_{5} I\right\}$,

$\Omega_{4,51}=\left[\begin{array}{ccccc}0 & 0 & 0 & 0 & 0 \\ Y_{1} & 0 & 0 & 0 & 0\end{array}\right]$,

$\Omega_{4,52}=\left[\Pi_{4,52}, \Pi_{4,52}, \Pi_{4,52}, \Pi_{4,52}\right]$,

$\Pi_{4,52}=\left[\begin{array}{ccc}\varepsilon_{6} B_{1}^{T} & 0 & \varepsilon_{6} I \\ 0 & 0 & 0\end{array}\right]$,

$\Omega_{4,53}=\left[\begin{array}{lll}0 & 0 & 0 \\ 0 & 0 & Y_{1}\end{array}\right], \quad \Omega_{4,54}=\left[\begin{array}{cc}0 & 0 \\ \varepsilon_{5} M_{2} & 0\end{array}\right]$,

$\Omega_{4,55}=\operatorname{diag}\left\{-\varepsilon_{6} I,-\varepsilon_{6} \delta_{2}^{2} I\right\}$.

Furthermore, the additive non-fragile $\mathrm{H} \infty$ controller gains in the form of (5) and (6) are $K_{0}=$ $Y_{0} X_{2}^{-1}(0,1)$ and $K_{1}=Y_{1} X_{1}^{-1}(1,1)$.

Proof. Consider the following Lyapunov functional $V[\bar{x}(k), \alpha(k)=i, \beta(k)=r]=\bar{x}^{T}(k) P(i, r) \bar{x}(k)$ where $P(i, r)=\operatorname{diag}\left\{P_{1}(i, r), P_{2}(i, r), P_{3}(i, r)\right\}$, $P_{1}(i, r), P_{2}(i, r)$ and $P_{3}(i, r)$ are symmetric positive definite matrices.

Taking the difference of $V[\bar{x}(k), \alpha(k)=i, \beta(k)$ $=r$ ] along the trajectory of system (9), we can obtain

$$
\begin{gathered}
\begin{aligned}
& \Delta V(k)= E\{V[\bar{x}(k+1), \alpha(k+1), \beta(k+1) \mid \bar{x}(k), \\
&\alpha(k)=i, \beta(k)=r]\}-V[\bar{x}(k), \alpha(k)=i, \\
&\beta(k)=r]=\eta^{T}(k) \Theta_{1}(i, r) \eta(k)
\end{aligned} \\
\text { where } \eta(k)=\left[\bar{x}^{T}(k), w^{T}(k), f^{T}(k, x(k))\right]^{T},
\end{gathered}
$$


$\Theta_{1}(i, r)=\left[\begin{array}{ccc}\Theta_{1,11}(i, r) & * & * \\ \bar{B}^{T} H \bar{A}(i, r) & \Theta_{1,22}(i, r) & * \\ \bar{F}^{T} H \bar{A}(i, r) & \bar{F}^{T} H \bar{B} & \bar{F}^{T} H \bar{F}-\varepsilon I\end{array}\right]$,

$\Theta_{1,11}(i, r)=\bar{A}^{T}(i, r) H \bar{A}(i, r)-P(i, r)+\varepsilon \Phi$,

$H=\sum_{j=0}^{1} \sum_{s=0}^{1} \lambda_{i j} \pi_{r s} P(j, s)$,

$\Theta_{1,22}(i, r)=\bar{B}^{T} H \bar{B}$.

Consider the following performance index

$J_{N}=E\left\{\sum_{k=0}^{N}\left[z^{T}(k) z(k)-\gamma^{2} w^{T}(k) w(k)\right]\right\}$.

Under zero initial condition, we can obtain

$$
\begin{aligned}
J_{N}= & E\left\{\sum _ { k = 0 } ^ { N } \left[z^{T}(k) z(k)-\gamma^{2} w^{T}(k) w(k)\right.\right. \\
& +\Delta V(k)]\}-E\left\{\sum_{k=0}^{N} \Delta V(k)\right\} \\
< & \sum_{k=0}^{N} \eta^{T}(k) \Theta_{2}(i, r) \eta(k)
\end{aligned}
$$

where

$$
\begin{aligned}
\Theta_{2}(i, r)= & \Theta_{1}(i, r)+[\bar{C}, \bar{D}, 0]^{T}[\bar{C}, \bar{D}, 0] \\
& -\left[0, \gamma^{2} I, 0\right]^{T}[0, I, 0] .
\end{aligned}
$$

Case 1. $i=0, r=0$. Let $X_{1}(i, r)=P_{1}^{-1}(i, r), X_{2}$ $(i, r)=P_{2}^{-1}(i, r), X_{3}(i, r)=P_{3}^{-1}(i, r), \varepsilon_{1}=\varepsilon^{-1}$, both sides of (11) are respectively multiplied by diag $\left\{\Lambda_{1,11}, \Lambda_{1,22}, \Lambda_{1,33}, \Lambda_{1,44}\right\}$, where $\Lambda_{1,11}=$ $\operatorname{diag}\{P(0,0), I, \varepsilon l\}, \Lambda_{1,22}=\operatorname{diag}\{I, I, I, I\}, \Lambda_{1,33}$ $=I, \Lambda_{1,44}=I$. By Schur complement, one can obtain $\Theta_{2}(0,0)<0$.

Case 2. $i=0, r=1$. Let $Y_{0}=K_{0} X_{2}(0,1)$, both sides of (12) are respectively multiplied by $\operatorname{diag}\left\{\Lambda_{2,11}, \Lambda_{2,22}, \Lambda_{2,33}, \Lambda_{2,44} \Lambda_{2,55}\right\}$, where $\Lambda_{2,11}=$ $\operatorname{diag}\{P(0,1), I, \varepsilon I\}, \Lambda_{2,22}=\operatorname{diag}\{I, I, I, I\}, \Lambda_{2,33}$ $=\operatorname{diag}\{I, I\}, \Lambda_{2,44}=\Lambda_{2,33}, \Lambda_{2,55}=\Lambda_{2,33}$. Using Lemma 1 and the method similar to case 1 , one can obtain $\Theta_{2}(0,1)<0$.

Case 3. $i=1, r=0$. By the method similar to Case 2, one can obtain $\Theta_{2}(1,0)<0$.

Case 4. $i=1, r=1$. Let $Q=\varepsilon_{4} P_{1}^{-1}(1,1), P_{1}^{-1}(1,1)$, $Y_{1}=K_{1} P_{1}^{-1}(1,1)$, by the method similar to Case 2 , one can obtain $\Theta_{2}(1,1)<0$.

According to the above, $\Theta_{2}(i, r)<0$ implies that matrix inequalities (10) hold. So the networked control system (9) is stochastically stable. On the other hand, as $N \rightarrow \infty$,

$J_{\infty}=E\left\{\sum_{k=0}^{\infty}\left[z^{T}(k) z(k)-\gamma^{2} w^{T}(k) w(k)\right]\right\}<0$.

Therefore, from definition 2 it follows that the networked control system (9) has an Hळo performance $\gamma$. This completes the proof.

Remark 1. The sufficient condition for the existence of multiplicative non-fragile $\mathrm{H} \infty$ controller in the form of (5) and (7) is similar to (11), (12), (13) and (14), except that $M_{2}$ and $N_{2}$ are substituted by $M_{3}$ and $N_{3} K_{\alpha(k)}$ respectively.

Remark 2. As the packet transmissions in both feedback and forward channels are considered simultaneously, the controller can be designed by the proposed algorithm. The obtained result is general.

Remark 3. The networked control system is modeled by augmented state, so the dimension of the system is enlarged, which makes the computation complex.

\section{Numerical Example}

To illustrate the effectiveness of the method proposed in this paper, consider the Lipschitz nonlinear system with the following parameters [6]

$$
\begin{aligned}
& A=\left[\begin{array}{ccc}
0.75 & -0.5 & 0 \\
0.7 & 0 & 0 \\
0 & 1 & 0
\end{array}\right], \quad B_{1}=\left[\begin{array}{l}
1 \\
0 \\
0
\end{array}\right], \quad B_{2}=\left[\begin{array}{l}
0.2 \\
0.2 \\
0.2
\end{array}\right], \\
& F=\left[\begin{array}{lll}
1 & 0 & 0 \\
0 & 1 & 0 \\
0 & 0 & 1
\end{array}\right], \quad f(k, x(k))=\left[\begin{array}{l}
0.03 \sin \left(x_{1}(k)\right) \\
0.01 \sin \left(x_{2}(k)\right) \\
0.02 \sin \left(x_{3}(k)\right)
\end{array}\right], \\
& w(k)=\left\{\begin{array}{l}
0,0 \leq k<30 \\
0.8,30 \leq k<60, C=[0.1,0,0], D=0.6 . \\
0, k \geq 60
\end{array}\right.
\end{aligned}
$$

The additive controller gain perturbations are $M_{2}=0.1$ and $N_{2}=[0.1,0.1,0.2]$, while the quantization densities of the quantizer of the system state and controller output are respectively $\rho_{1}$ $=0.5$ and $\rho_{2}=0.6$. The transition probability matrices of Markov chains are 


$$
\bar{P}=\left[\begin{array}{ll}
0.2 & 0.8 \\
0.4 & 0.6
\end{array}\right], \quad \bar{Q}=\left[\begin{array}{ll}
0.3 & 0.7 \\
0.2 & 0.8
\end{array}\right] .
$$

The H $\infty$ performance is $\gamma=1.5$.

The packet transmissions in the communication channels from the sensor to the controller and from the controller to the actuator are shown in Figure 2 and Figure 3 respectively.

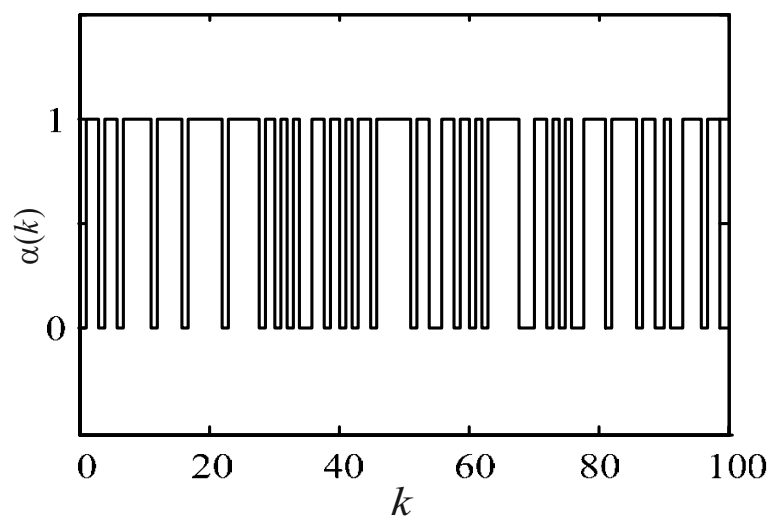

Figure 2. Packet transmission from sensor to controller.

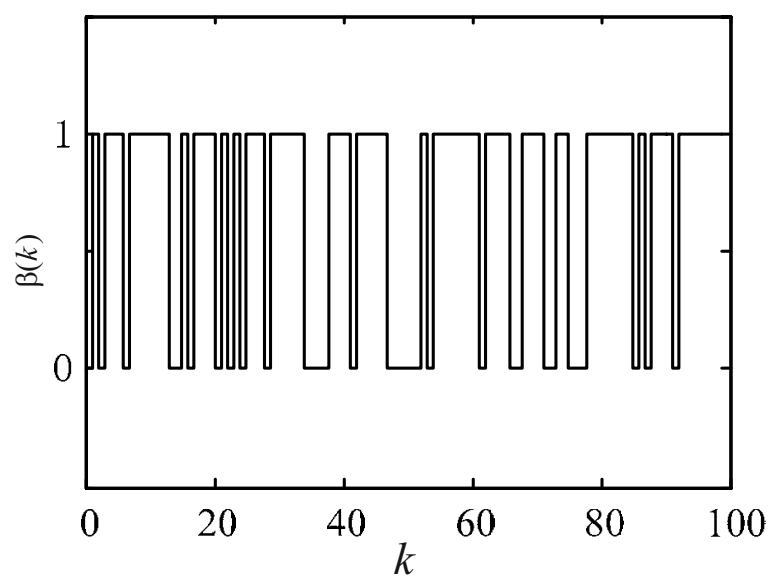

Figure 3. Packet transmission from controller to actuator.

Solving the inequalities (11), (12), (13) and (14) by the MATLAB linear matrix inequality toolbox, we can obtain the additive networked non-fragile $\mathrm{H} \infty$ controllers

$$
\begin{aligned}
& K_{0}=[-0.0099,-0.0099,-0.0198], \\
& K_{1}=[-0.0122,0.0135,-0.0004] .
\end{aligned}
$$

When the packet dropouts are not taken into consideration, the existent condition of the clas- sical controller is reduced to linear matrix inequality (14). Solving the inequality (14) by the MATLAB linear matrix inequality toolbox, we can obtain the additive networked non-fragile $\mathrm{H} \infty$ controllers

$$
K=[-0.4093,-0.4436,-0.4112] \text {. }
$$

Assume that the initial state of the system is $[1.5,0,-1]^{T}$. The state trajectories of the networked control system controlled by the proposed and classical controllers are shown in Figure 4 - Figure 6 . It can be seen that the system is stable. The dynamic and static performance indices of the proposed controller are superior to those of the classical controller, which verifies the effectiveness of the method presented in this paper.

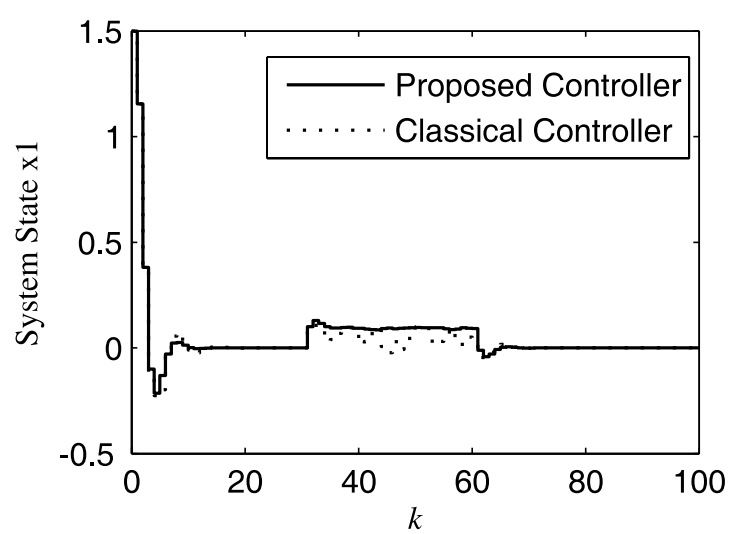

Figure 4. Trajectories of state $\mathrm{x} 1$ of networked control system.

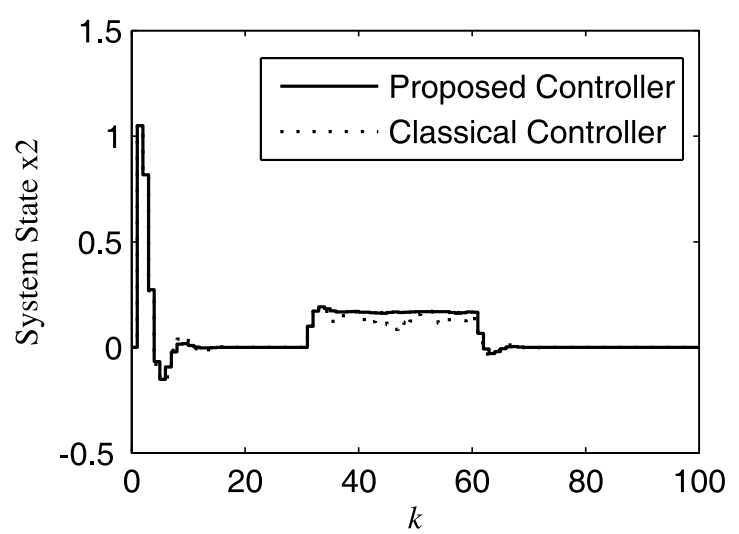

Figure 5. Trajectories of state $\mathrm{x} 2$ of networked control system. 


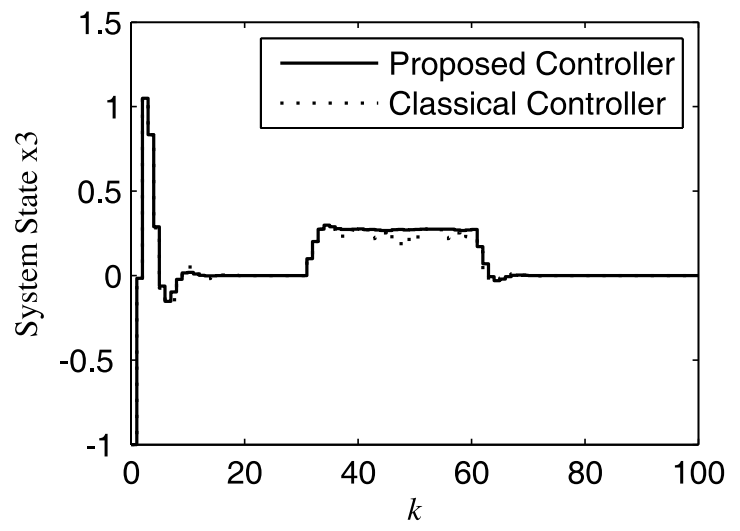

Figure 6. Trajectories of state $\mathrm{x} 3$ of networked control system.

\section{Conclusion}

In this paper, the networked non-fragile $\mathrm{H} \infty$ controller for Lipschitz nonlinear system is designed. The logarithmic quantizer and packet dropouts governed by Markov chain in the feedback and forward communication channels are taken into consideration simultaneously. The sufficient condition established for the existence of non-fragile $\mathrm{H} \infty$ controller is general. If the packet dropouts are not considered, the existent condition of the classical controller is simplified to linear matrix inequality. The controller can be obtained by solving a set of linear matrix inequalities. A simulation example is presented to demonstrate the effectiveness and superiority of the method proposed in this paper.

\section{Acknowledgements}

The authors would like to thank the editor and the anonymous reviewers for the helpful suggestions. This work was supported by the National Natural Science Foundation of China under grants 51307089.

\section{References}

[1] R. A. Gupta and M. Y. Chow, "Networked Control System: Overview and Research Trends", IEEE Transactions on Industrial Electronics, vol. 57, no. 7, pp. 2527-2535, 2010. http://dx.doi.org/10.1109/tie.2009.2035462
[2] Z. D. Wang et al., "Quantized Hoo Control for Nonlinear Stochastic Time-delay Systems with Missing Measurements", IEEE Transactions on Automatic Control, vol. 57, no. 6, pp. 1431-1444, 2012.

http://dx.doi.org/10.1109/tac.2011.2176362

[3] F. Rasool et al., "Robust Ho Output Feedback Control of Discrete-time Networked Systems with Limited Information", Systems \& Control Letters, vol. 60, no. 10, pp. 845-853, 2011. http://dx.doi.org/10.1016/j.sysconle.2011.07.001

[4] Y. Shi and B. Yu, "Output Feedback Stabilization of Networked Control Systems with Random Delays Modeled by Markov Chains", IEEE Transactions on Automatic Control, vol. 54, no. 7, pp. 1668-1674, 2009. http://dx.doi.org/10.1109/tac.2009.2020638

[5] Z. D. Wang et al., "Robust $\mathrm{H} \infty$ Control for Networked Systems with Random Packet Losses", IEEE Transactions on Systems, Man and Cybernetics, Part B (Cybernetics), vol. 37, no. 4, pp. 916-924, 2007. http://dx.doi.org/10.1109/tsmcb.2007.896412

[6] M. Liu et al., "State Estimation and Stabilization for Nonlinear Networked Control Systems with Limited Capacity Channel", Journal of the Franklin Institute, vol. 348, no. 8, pp. 1869-1885, 2011. http://dx.doi.org/10.1016/j.jfranklin.2011.05.008

[7] J. L. Xiong and J. Lam, "Stabilization of Linear Systems Over Networks with Bounded Packet Loss", Automatica, vol. 43, no. 1, pp. 80-87, 2007. http://dx.doi.org/10.1016/j.automatica.2006.07.017

[8] P. Seiler and R. Sengupta, "An Ho Approach to Networked Control", IEEE Transactions on Automatic Control, vol. 50, no. 3, pp. 356-364, 2005. http://dx.doi.org/10.1109/tac.2005.844177

[9] L. H. Keel and S. P. Bhattacharyya, "Robust, Fragile, or Optimal?", IEEE Transactions on Automatic Control, vol. 42, no. 8, pp. 1098-1105, 1997. http://dx.doi.org/10.1109/9.618239

[10] R. Q. Lin et al., "Non-fragile Guaranteed-cost Controller Design for the Networked Control Systems with Network-induced Time Delay", in 2010 IEEE International Conference on Automation and Logistics, 2010, pp. 614-617. http://dx.doi.org/10.1109/ical.2010.5585358

[11] Y. Zhang et al., "Non-fragile Control for Nonlinear Networked Control Systems with Long Time-delay", Computers \& Mathematics with Applications, vol. 57, no. 10, pp. 1630-1637, 2009. http://dx.doi.org/10.1016/j.camwa.2009.03.053

[12] Z. Zhang et al., "Optimal Robust Non-fragile $\mathrm{H} \infty$ Control for Networked Control Systems with Uncertain Time-delays", in Proceedings of the 11th World Congress on Intelligent Control and Auto- 
mation, 2014, pp.4076-4081.

http://dx.doi.org/10.1109/wcica.2014.7053398

[13] N. Xie and B. Xia, "Non-fragile Guaranteed Cost Fault Tolerant Control for Networked Control Systems with Fast-varying Delay", in 2010 3rd International Conference on Computer Science and Information Technology, 2010, pp. 396-400. http://dx.doi.org/10.1109/iccsit.2010.5564837

[14] J. W. Zhu et al., "Non-fragile Hळ Control for Networked Control Systems with Quantization and Time-delays", in 2011 Chinese Control and Decision Conference (CCDC), 2001, pp. 4276-4280. http://dx.doi.org/10.1109/ccdc.2011.5968260

[15] Z. N. Li et al., "Non-fragile $\mathrm{H} \infty$ Control with Randomly Occurring Gain Variations, Distributed Delays and Channel Fadings", IET Control Theory \& Applications, vol. 9, no. 2, pp.222-231, 2015.

http://dx.doi.org/10.1049/iet-cta.2014.0426

[16] Z. N. Li et al., "Non-fragile Hळ Control with Randomly Occurring Gain Variations", in 2014 20th International Conference on Automation and Computing, 2014, pp.9-13.

http://dx.doi.org/10.1109/iconac.2014.6935452

[17] Y. Liu and L. Y. Sun, "Non-fragile Control for a Class of Networked Switch Fuzzy Time-delay Systems", in 2010 International Conference on Machine Learning and Cybernetics, 2010, pp. 932-936.

http://dx.doi.org/10.1109/icmlc.2010.5580605

[18] F. L. Zhu and Z. Z. Han, "A Note on Observers for Lipschitz Nonlinear System", IEEE Transactions on Automatic Control, vol. 47, no. 10, pp. 1751-1754, 2002.

http://dx.doi.org/10.1109/TAC.2002.803552

[19] M. Y. Fu and L. H. Xie, "The Sector Bound Approach to Quantized Feedback Control", IEEE Transactions on Automatic Control, vol. 50, no. 11, pp. 1698-1711, 2005.

http://dx.doi.org/10.1109/tac.2005.858689

[20] P. Shi et al., "Robust Control for Markovian Jumping Discrete-time Systems", International Journal of Systems Science, vol. 30, no. 8, pp. 787-797, 1999.

http://dx.doi.org/10.1080/002077299291912

[21] L. H. Xie, "Output Feedback Hळ Control of Systems with Parameter Uncertainty", International Journal of Control, vol. 63, no. 4, pp. 741-750, 1996.

http://dx.doi.org/10.1080/00207179608921866
Received: August 2016

Revised: September 2017

Accepted: October 2017

Contact addresses:

Weiguo Ma

College of Electrical Engineering

Nantong University

Nantong, Jiangsu, 226019

China

e-mail: wgma@ntu.edu.cn

$\mathrm{Xia} \mathrm{Xu}$

College of Electrical Engineering

Nantong University

Nantong, Jiangsu, 226019

China

e-mail: xuxia@ntu.edu.cn

Hairong Zhu

College of Electrical Engineering

Nantong University

Nantong, Jiangsu, 226019

China

e-mail: zhu.hr@ntu.edu.cn

WeIguo MA received his $\mathrm{PhD}$ degree in control science and engineering from Dalian University of Technology. He is currently an associate professor at the College of Electrical Engineering, Nantong University, China. His research interests include robust control and networked control.

$\mathrm{XIA}_{\mathrm{IA}} \mathrm{X}$ received her MSc degree in electrical engineering from Shanghai University. She is currently a lecturer at the College of Electrical Engineering, Nantong University, China. Her research interests include intelligent building and electrical equipment.

HAIRONG ZHU received his PhD degree in control science and engineering from Southeast University. He is currently an associate professor at the College of Electrical Engineering, Nantong University, China. His research interests include adaptive control and intelligent robot systems. 